\title{
Des imaginaires pour se projeter ou pour critiquer? Réflexions méthodologiques
}

\section{Projecting or criticizing imaginaries? Methodological reflections}

\author{
Nicolas Minvielle ${ }^{1}$, Remy Hemez ${ }^{2}$, Olivier Wathelet ${ }^{3}$ \\ ${ }^{1}$ Audencia Business School, France, nminvielle@audencia.com \\ ${ }^{2}$ Officier de l'armée de Terre, France, rhemez@yahoo.fr \\ ${ }^{3}$ Users Matter, Belgique, olivier@users-matter.eu
}

RÉSUMÉ. A travers l'exemple du fantassin, l'article propose des outils méthodologiques pour exploiter des imaginaires de la science-fiction (film, manga, roman, bédé, ...) dans une démarche de conception. Sur base d'un corpus de près de 300 références, il montre que le recours aux imaginaires doit faire l'objet d'un traitement différentié selon le support dont il est question, et qu'il est possible de distinguer des enjeux de contexte et des enjeux d'usage qui sont complémentaires. L'article expose différentes formes d'enjeux stratégiques et compare les solutions proposées dans ces imaginaires avec celles ayant cours dans les champs de l'industrie militaire. En conclusions, l'article discute des enjeux de circulation des imaginaires entre industries culturelles et militaires.

ABSTRACT. Through the example of the infantryman, this article proposes methodological tools to exploit imaginaries from science fiction (film, manga, novel, comics, etc.) in a design approach. Based on a corpus of nearly 300 references, it shows that the imaginaries should be used in a differentiated way according to the medium that is considered and that it is possible to distinguish complementary context issues and use issues. The article discusses the different forms of strategic issues and compares the solutions proposed in these imaginaries with those taking place in the fields of the military industry. In conclusion, the article discusses issues concerning the circulation of imaginaries between cultural and military industries.

MOTS-CLÉS. Science-fiction, culture populaire, design fiction, militaire, méthodologie.

KEYWORDS. Science fiction, pop culture, design fiction, military, methodology.

\section{Introduction}

Marginal au sein des pratiques managériales il y a 5 ans encore, le design fiction - démarche spéculative du design visant à concevoir des mondes potentiels pour, sur un mode généralement dystopique, susciter une réflexion avancée sur ses conséquences pour les choix contemporains - est aujourd'hui employé et enseigné dans de nombreuses institutions, en particulier dans le cadre de réflexions stratégiques. Peu de recherches, toutefois, s'interrogent sur l'efficacité de cette démarche, qu'il s'agisse de ses effets sur un collectif en terme de projection, de débat ou encore d'acquisition de compétences de résiliences, pour citer 3 bénéfices abordés dans la littérature spécialisée [AUG 13] [COU 18] [MIN 15].

Dans cet article, nous souhaitons exposer des réflexions issues d'une recherche en cours portant en particulier sur la contribution des imaginaires de la culture populaire à l'activité de projection de visions crédibles du futur. Les imaginaires sont ici définis comme des représentations d'un futur sous une forme qui peut être figurative ou narrative. A l'aide de l'exemple du fantassin - richement représenté dans les œuvres de science-fiction (romans, bande dessinée, films) - nous souhaitons explorer différents leviers permettant d'exploiter cette matière pour générer des propositions d'avenir crédibles.

La perspective de recherche que nous exposons ici s'articule sur deux hypothèses.

- La première est que les imaginaires mobilisés sont par nature de qualités diverses, et que leurs capacités projectives le sont tout autant. 
- La seconde hypothèse méthodologique que nous formulons est que l'on peut distinguer différents niveaux de focalisation des imaginaires, en raison des choix créatifs de leurs concepteurs, ce qui oriente le travail d'appropriation. Cette variation d'échelle se focalisera sur au moins deux niveaux que nous proposons d'interroger: les usages de technologies « imaginaires » en tant que laboratoires pour tester leur fonctionnement; et la mise en scène d'évolutions de l'environnement sociotechnique qui offrent la possibilité d'appréhender de manière créative les possibles transformations du monde.

Dans cet article, essentiellement tourné vers des questions méthodologiques donc, nous montrerons comment un large corpus d'imaginaires peut être appréhendé à ces différentes échelles. Nous exposerons d'abord les différentes technologies relatives aux fantassins déployées dans la littérature, montrant l'existence de déséquilibres manifestes dans les formes d'augmentations décrites. Nous montrerons que celles-ci sont loin de se montrer fantaisistes, mais répondent à des problématiques réellement étudiées par les professionnels du secteur. Nous verrons également que les scènes décrites dans le cas de situations de guerre potentielles - à travers l'exemple des nouvelles vulnérabilités permettent en effet de pointer du doigt des enjeux réels complémentaires des approches contemporaines.

La seconde partie de notre exposé sera consacrée à la nature des composantes fictionnelles pertinentes à engager dans une démarche de design fiction: du contexte et des usages décrits. Cette dernière piste s'avère certainement la plus féconde, car elle offre l'occasion de tester des implications possibles de choix d'interactions, analyse très précieuses au sein des démarches de conception. A ce titre, les « erreurs de prédictions » et interactions dont le caractère problématique « saute aux yeux » du spectateur constituent aussi de bons laboratoires d'idées pour considérer le développement d'une solution technique nouvelle.

Notre propos est ainsi d'explorer la façon dont les imaginaires de la science-fiction peuvent être utilisés concrètement par des concepteurs pour spéculer raisonnablement sur le futur de solutions techniques. Quelques indices montrent que cette pratique de spéculation existe d'ores et déjà, ce dont nous parlerons rapidement en conclusion de cet article.

\section{Enjeux de la production d'un corpus d'imaginaires}

Dans le cadre de notre projet, nous avons créé une base de données de près de 300 imaginaires portant sur le combattant débarqué. Quatre principaux mediums ont été sollicités : les films et dessins animés ; la bande dessinée et le manga ; les romans et nouvelles ; et les jeux vidéo. A l'exception des romans et nouvelles, dont la diversité et le volume de publication en font des ressources évidentes, les autres sources sont choisies en raison du caractère visuel des imaginaires, Les participants aux ateliers que nous conduisons lors de séances de debriefing estiment en effet qu'elles permettent une appropriation rapide d'une idée complexe et qu'elles rendent possibles des projections multiples. Nous proposons de relier cette double propriété aux enjeux de notre première hypothèse, à savoir la capacité de coupler ouverture et cohérence, invitant à questionner la nature du support dans cet équilibre.

Pour créer ce corpus, nous sommes allés en librairies et magasins spécialisés, puis nous avons approfondi en consultant notamment des sites de fans ou des encyclopédies spécifiques. De nombreux jeux vidéo disposent ainsi de leur wiki où des fans détaillent de manière extrêmement précise les capacités des armes, armures et équipements divers. ${ }^{1}$ Il en va de même pour les animes et les mangas ${ }^{2}$. Les super héros de l'univers Marvel ou DC Comics disposent aussi d'analyses proposées sur des sites

\footnotetext{
${ }^{1}$ Wiki de Starcraft présentant le CMC Powered Combat Suit, par exemple.

${ }^{2}$ Super Robot Wars Wiki présentant Tekkaman Blade,par exemple. 
tels que Comic Vine et d'encyclopédies détaillant leurs pouvoirs, augmentations et faiblesses, ce qui les rend plus accessibles. Ce travail repose sur une forte serendipité.

A noter ici que l'usage des bases de données ne permet pas d'accéder à des niveaux de détails pertinents pour la collecte des imaginaires lorsqu'il s'agit de ressources visuelles, et le traitement automatique des données livresques reste encore à construire pour générer des filtres efficaces. Cette part de l'humain, et notamment de l'expertise, reste, selon nous, la meilleure solution, quoique chronophage et par nature incertaine.

Nous avons ensuite évalué douze critères allant de la typologie d'assistance cognitive aux améliorations offertes, en passant par la capacité des fantassins à travailler en groupe, ou encore le milieu dans lequel ils sont censés opérer.

Ces données quantitatives ont ensuite été mises en perspective par une analyse qualitative des imaginaires, en nous intéressant notamment à la façon dont les concepteurs eux-mêmes avaient envisagé leurs créations. Ce dernier point est parfois mis en avant très clairement dans les biographies d'auteurs ou dans les notes de bas de page d'œuvres fameuses, à l'instar de la réédition récente de Ghost In the Shell accompagnée des commentaires de son auteur principal. Des captures d'écran de films ou des scans de bandes dessinées nous ont ainsi permis de souligner certains éléments, et de les confronter avec l'expérience du réel d'un des co-auteurs, lieutenant-colonel dans l'armée de Terre.

De ces différentes approches, nous pouvons tirer quatre principaux éléments de méthode. Tout d'abord le fait que parmi cette masse d'imaginaires, tous ne sont pas de même qualité. Pour autant, nombre d'entre eux ont démontré une réelle capacité à représenter de manière extrêmement réaliste des enjeux actuels. Ensuite, et c'est peut-être le plus intéressant, ils nous offrent aussi des visions très abouties des enjeux de demain qu'il devient possible de tester et d'éprouver. Finalement, se pose la question de savoir comment utiliser ce terreau de créativité de manière intelligente en distinguant contexte et usage.

\section{Des imaginaires de qualités diverses}

Le premier enjeu de notre démarche réside dans la collecte des imaginaires en tant que telle.Certains se sont en effet révélés plus riches que d'autres. Les comics, par exemple, proposent des super héros disposant de pouvoirs magiques leur permettant de voler ou de devenir invisibles. S'il est intéressant de compiler de manière quantitative ces pouvoirs et de souligner que le fait de voler est la capacité la plus récurrente (voir tableau ci-dessous), lorsque l'on regarde comment les imaginaires nous proposent de faire voler les héros une explication «magique » n'est pas d'une grande utilité pour le monde réel.

\begin{tabular}{|c|c|}
\hline $\begin{array}{c}\text { Types } \\
\text { d'améliorations }\end{array}$ & $\begin{array}{c}\text { \% D'occurrences } \\
\text { dans les imaginaires }\end{array}$ \\
\hline Agilité & $43,55 \%$ \\
\hline $\begin{array}{c}\text { Amélioration } \\
\text { de la vue }\end{array}$ & $20,43 \%$ \\
\hline $\begin{array}{c}\text { Amélioration } \\
\text { de l'écoute }\end{array}$ & $19,35 \%$ \\
\hline $\begin{array}{c}\text { Amélioration } \\
\text { de la vitesse et } \\
\text { de l'endurance }\end{array}$ & $6,45 \%$ \\
\hline
\end{tabular}

Tableau 1. Les cinq capacités physiques les plus améliorées dans les imaginaires de notre corpus de 289 œuvres de fiction 


\begin{tabular}{|c|c|}
\hline $\begin{array}{c}\text { Types } \\
\text { d'améliorations }\end{array}$ & $\begin{array}{c}\text { \% D'occurrences dans } \\
\text { les imaginaires }\end{array}$ \\
\hline Voler & $52,26 \%$ \\
\hline Sauter & $24,52 \%$ \\
\hline Se cacher & $23,23 \%$ \\
\hline
\end{tabular}

Tableau 2. Les trois améliorations les plus évoquées dans les imaginaires de notre corpus de 289 œuvres de fiction

A l'inverse, les jeux vidéo ou certains mangas et dessins animés offrent des visions extrêmement précises de la manière dont les capacités offertes aux combattants fonctionnent. Dans Call of Duty, la capacité à devenir invisible est proche de ce que l'on peut voir actuellement dans des systèmes tels que ceux développés par BAE. Par ailleurs, cette capacité est rendue très réaliste par le fait qu'elle utilise énormément de batterie et a donc une durée d'usage très limitée. Entre ces deux extrêmes, on se rend compte que certains supports offrent plus de potentiels que d'autres. Les jeux vidéo, par leur volonté de réalisme, offrent une vision du futur radicale mais qui tente d'être réaliste, et donc pseudo-justifiée scientifiquement. Les comics à l'inverse prennent plus de libertés sur le sujet.

Si les imaginaires sont donc très divers en termes de richesse, ceux qui sont pertinents offrent cependant des visions extrêmement riches.

\section{Des représentations souvent appropriables et exploratoires}

Une première exploitation de notre base de données s'est attachée à identifier les différentes façons dont les combattants sont «augmentés $»^{3}$, que ce soit via la technologie ou la «physiologie » (amélioration des capacités physiques et cognitives du combattant).

Voler arrive largement en tête des augmentations. C'est un élément classique des projections de mobilité, en témoignent les imaginaires et les projets de voitures volantes, presque aussi anciens que les voitures elles-mêmes ${ }^{4}$. Si le vol individuel pour le combattant, un projet datant du début des années $1950^{5}$, ne paraît pas envisageable à court terme, le sujet revient régulièrement, à l'instar des prototypes de Flyboard Air de la société française Zapata Racing qui a récemment attiré l'attention de la DARPA américaine et des forces spéciales françaises.

La recherche d'augmentation de la force est également récurrente (via, notamment, de performants exosquelettes), immédiatement suivie d'un accroissement de l'agilité et de la vision. Il s'agit de compenser les limites physiques et physiologiques de l'homme au combat. Prenons simplement l'exemple de la fatigue. Une étude menée pendant 20 jours sur des unités d'artillerie de l'US Army a montré que celles disposant de sept heures de sommeil quotidien avaient une efficacité très proche de $100 \%$. En revanche, celles qui ne dormaient que quatre heures perdaient $15 \%$ d'efficacité et devenaient même dangereuses [GRO 08]. D'où l'intérêt ancien pour la psychopharmacologie : caféine et cocaïne, au départ, puis amphétamines et modafinil (qui permettrait de rester en alerte 40 heures) afin de maintenir éveillé. 35 millions de comprimés de méthamphétamines auraient été utilisés durant la Blitzkrieg entre avril et juin 1940 [RAS 08]. Cette approche - et les risques qu'elle induit - apparaît aussi régulièrement dans les imaginaires. Les mercenaires du comic américain Nash (1999) qui prennent régulièrement de la drogue sont fuis par les populations civiles qui craignent leur impulsivité après injection.

\footnotetext{
${ }^{3}$ Sur la question du soldat augmenté, se référer, notamment au n ${ }^{\circ} 32$ de la revue Inflexions : « Le soldat augmenté ? », 2016.

${ }^{4}$ À titre d'exemple, deux projets actuels de voiture volante : l'Aeromobil 4.0 STOL (https://www.aeromobil.com/aeromobil-4 0stol/) et le PAL-V Liberty (https://www.pal-v.com/).

${ }^{5}$ Le Piasecki VZ-8 Airjeep était, par exemple, un prototype d'aérogire développé par l'armée américaine entre 1957 et 1959. 
Certains des imaginaires se révèlent donc particulièrement intéressants du point de vue projectif en offrant des représentations claires des enjeux actuels et à venir du combattant débarqué. Pour reprendre l'exemple du camouflage et de l'invisibilité utilisée dans les jeux vidéo, des travaux développent depuis plusieurs années [SHU 06] des métasurfaces diélectriques permettant de rendre invisibles leurs porteurs (robot, drone, véhicule, voire combattant) à certaines longueurs d'ondes (jusqu'à $70 \%$ d'absorption pour les ondes radars, même la lumière visible est concernée). Des projets de camouflage s'appuyant sur ces découvertes sont déjà relativement aboutis comme le Quantum Stealth de la société canadienne Hyperstealth, dont des prototypes ont été présentés en $2016^{6}$. Il en va de même pour la capacité à voler, quelques prototypes issus de la société civile étant analysés par les forces armées pour évaluer leur potentiel.

Ghost in the Shell, permet, lui, entre autres, d'aborder la thématique du cyber. Depuis 1989, l'auteur, très informé de la chose militaire et des évolutions technologiques y propose un travail qui, par de nombreux aspects, pourrait être visionnaire, nous renvoie à des conceptions actuelles de la guerre du futur, et permet de les interroger. Par exemple, dans le manga, la maitrise cyber est utilisée avant, pendant ou après une attaque physique, mais toujours de manière intrinsèquement liée. Avant une offensive physique, l'attaque cyber peut avoir pour rôle de semer la confusion dans les troupes adverses, voire de neutraliser certains éléments.

Les militaires le savent bien, tout combat devrait désormais être précédé d'une lutte pour acquérir la supériorité dans l'espace électromagnétique et cyber. Tâche extrêmement difficile étant donné les caractéristiques de ce milieu «lisse » (architecture ouverte et étendue). Pendant une attaque physique, le hacking des capteurs d'une zone dans laquelle le major doit intervenir lui permet de dérouter les combattants ennemis. Face à un cyborg armé d'une mitrailleurs Gatling, elle peut avancer droit sur lui, ayant piraté son interface de visée. Après une attaque physique, il s'agit souvent pour les combattants de prendre le contrôle d'un centre de stockage ou de traitement de données ou de se connecter sur les machines de l'ennemi pour l'infiltrer davantage. On voit là les prémisses d'une nouvelle forme d'exploitation d'une offensive. Cela souligne aussi la question de la sécurité d'un réseau si une ou plusieurs machines sont saisies par les adversaires. Bien entendu, des boutons d'effacement d'urgence existent sur les postes radios mais les soldats seront-ils toujours en mesure de les utiliser ? N'est-il pas possible de récupérer des données même après effacement ?

\section{De nouvelles vulnérabilités}

Certaines fictions sont encore plus intéressantes car elles invitent à regarder l'envers des innovations, en pointant du doigt leurs vulnérabilités et en proposant une description fine des usages. On ne parle pas de «voler » en général, mais de découvrir un mode d'emploi d'exosquelette en pleine bataille, situation particulièrement dangereuse (cf. le running gag au début du film The Edge of Tomorrow, 2014). Dans notre corpus, la vulnérabilité la plus représentée consiste à détruire les «augmentations » d'un ennemi grâce à une impulsion électromagnétique ou à une surcharge électrique. On en trouve un bel exemple dans le film Pacific Rim (2013) où un Kaiju «électrocute » un Jaeger et fait écho aux développements actuels d'armes électromagnétiques non-nucléaires (e-bomb). Les deux autres vulnérabilités les plus évoquées sont, dans l'ordre, le défaut d'énergie et le hacking.

\footnotetext{
${ }^{6}$ Ces matériaux sont cependant très difficiles à manufacturer et demandent une grande maitrise des nanotechnologies. 


\begin{tabular}{|c|c|}
\hline Type de faiblesse & $\begin{array}{c}\text { \% D'occurrence } \\
\text { dans les } \\
\text { imaginaires }\end{array}$ \\
\hline $\begin{array}{c}\text { Attaques } \\
\text { électromagnétiques }\end{array}$ & $14 \%$ \\
\hline $\begin{array}{c}\text { Dépendance à sa } \\
\text { source d'énergie }\end{array}$ & $14 \%$ \\
\hline Hacking & $14 \%$ \\
\hline $\begin{array}{c}\text { Lenteur du } \\
\text { déplacement }\end{array}$ & $11 \%$ \\
\hline $\begin{array}{c}\text { Exposition de } \\
\text { parties vitales }\end{array}$ & $9 \%$ \\
\hline
\end{tabular}

Tableau 3. Les dix vulnérabilités les plus évoquées dans les imaginaires de notre corpus de 289 œuvres de fiction

Sur ce dernier point, les séries Apple Seed (1985) et Ghost in the shell (1989) étaient très en avance. Quasiment tous les combats de ces deux productions japonaises impliquent du hacking. L'héroïne de Ghost in the Shell peut se connecter physiquement à tout système informatique et «plonger» dans ses entrailles. Dans Ghost in the Shell the new movie (2017), la vue d'un tireur ennemi est piratée pour l'empêcher de viser correctement. Cela rejoint parfaitement les préoccupations des militaires qui, lorsqu'il s'agit d'évoquer l'environnement opérationnel futur et en s'appuyant sur les retours d'expérience des conflits actuels (Ukraine, en particulier), estiment souvent que tout combat sera précédé d'une phase de «conquête » de l'environnement électromagnétique et cyber [HUB 00]. Le film Kill Command (2016) offre quant à lui une lecture critique des risques cybers liés à l'usage de composants électroniques étrangers ${ }^{7}$. On y voit un sous-traitant industriel se connecter au fusil d'un sniper, obtenir l'accès au software et le débrancher. La décision du Pentagone de mai 2018 de suspendre l'acquisition et l'utilisation de drones commerciaux à cause des questions de sécurité opérationnelle s'inscrit tout à fait dans ce cadre [ATH 18].

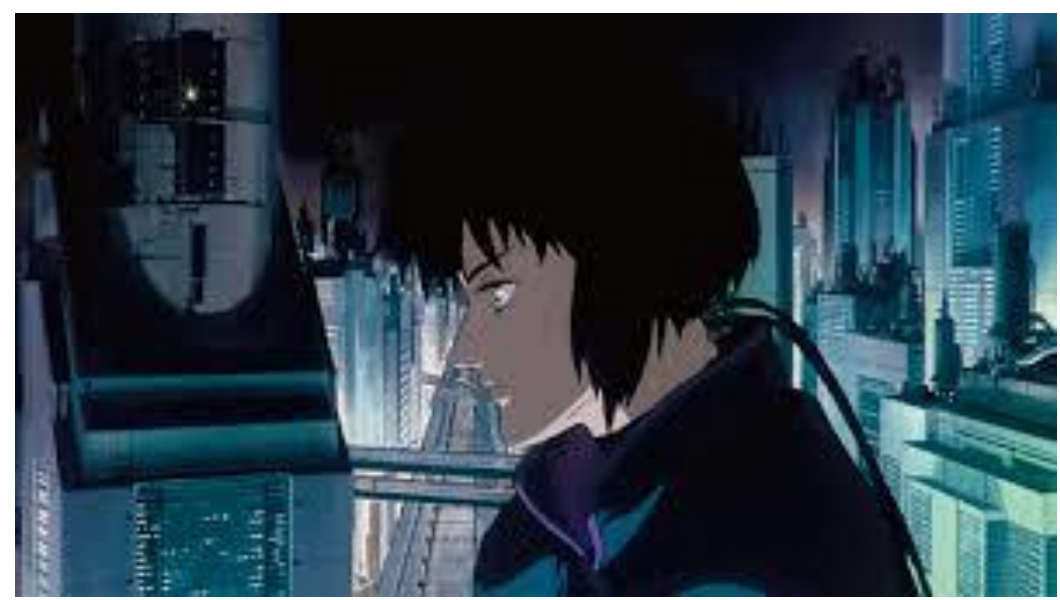

Figure 1. Ghost in the Shell

\footnotetext{
${ }^{7}$ Un risque également présent dans [SIN 15] 


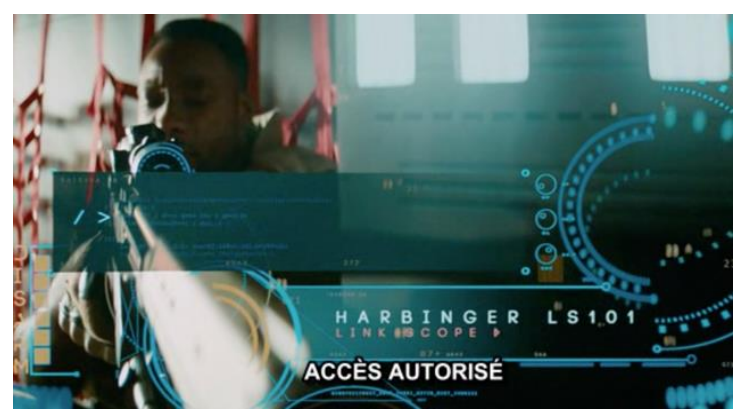

Figure 2. Kill Command

Comme les deux parties précédentes l'ont détaillé, les imaginaires arrivent à mettre en perspective les enjeux actuels, tout en présentant ceux de demain, en offrant des visions d'usages nouveaux, ou de technologies nouvelles, ainsi que leurs limitations. Se pose maintenant la question de savoir comment appréhender ces enjeux plus concrètement, en fonction des contextes ou des usages présentés.

\section{Le contexte, un premier point d'entrée pour s'approprier les imaginaires}

Par contexte, nous entendons la capacité qu'ont les imaginaires de rendre compte de situations de manière crédible, ouvertes, et à ce titre exploitable pour interroger les projections actuelles qui sont supposées les adresser. Dans le domaine du combat débarqué que nous utilisons ici comme exemple, on peut illustrer ce thème par la problématique de l'augmentation, qu'elle soit physique ou cognitive.

Dans les imaginaires, les combattants ayant subi d'importantes augmentations ont souvent tendance à se reposer abusivement sur elles et à oublier les fondamentaux du combat. Apple Seed offre aussi la vision d'une héroïne humaine, Deunan Knute, qui dispose d'une expérience telle qu'elle est en mesure de prendre le dessus dans ses combats avec des cyborgs, en particulier par la ruse. Dans une scène du film Apple Seed Ex Machina (2007), elle aveugle des preneurs d'otages avec une grenade, ce qui lui permet de sauver les captifs. Toujours dans la même série, en collaboration avec son partenaire (qui est un cyborg), elle doit se débarrasser de «mines robots autonomes » particulièrement dangereuses. Elle y parvient en combinant un appât, son partenaire qui s'avance vers elle, et des tirs à longue distance. On est donc loin de la vision caricaturale d'une suprématie des machines.

Ces imaginaires permettent ainsi d'interroger le concept de supériorité technologique. On y trouve une critique récurrente de la «tentation cybernétique à outrance ». Les personnages ayant subi trop d'améliorations ont souvent tendance à se reposer abusivement sur elles et à en oublier les fondamentaux du combat. Dans l'excellent Ghost in the Shell - qui dès 1989, floutait la frontière entre humains et machines, dotant les premiers d'atouts cybernétiques et les deuxièmes d'intelligence - le seul personnage majeur totalement humain de cette série, Togusa, réussit à égaler au combat nombre de ses camarades augmentés ou même totalement cyborgs. Cette résilience de l'humain, avec ses capacités spécifiques, nous éloigne de la vision caricaturale d'une suprématie totale des machines telle qu'elle a pu être véhiculée par d'autres fictions, comme Terminator.

Cela doit nous interroger sur la supériorité technologique d'une armée. Il est souvent dit que la diffusion des technologies duales amenuise sa supériorité opérationnelle. Il convient cependant de souligner que l'accélération de la propagation de savoir-faire tactiques vers et entre les groupes irréguliers est une menace toute aussi sérieuse : «l'élément le plus novateur de ces dernières années réside plutôt dans l'élargissement de la capacité à produire des soldats. » [GOY 16] Boko Haram a, par exemple, démontré sa capacité à coordonner des manœuvres relativement complexes combinant appuifeu et mouvement. La bataille de Mossoul, en dépit d'un rapport de force de 20 contre 1, a mis en évidence les capacités tactiques de l'EI, avec notamment la combinaison de moyens de renseignement, de frappe indirecte, de contre-mobilité et de manœuvre. Les adversaires non-étatiques disposent, de plus, d'une capacité d'adaptation permanente leur permettant d'intégrer plus rapidement à leurs modes 
d'action les technologies disponibles. Le cas des drones artisanaux est symptomatique de cette tendance. A moyens égaux, il est possible d'être plus efficaces avec de l'imagination (importance des innovations organisationnelles et doctrinales).

De plus, les imaginaires nous invitent à envisager un avenir « analogique » du combattant, moins dépendant des technologies et moins détectable du fait de son faible «rayonnement» électromagnétique. Le futur du soldat est sans doute aussi low-tech [COK 15]. Enfin, les imaginaires peuvent offrir des cas concrets pour réfléchir sur la distance entre la représentation virtuelle de la guerre au sol, ou près du sol et sa réalité actuelle et future. Trop souvent ${ }^{8}$, les équipements militaires sont envisagés en dehors de tout combat rapproché. Or, cette vision de la guerre «stand off» et de haute technologique est probablement une «banqueroute " ${ }^{9}$, à cause de son coût, et parce qu'elle ne suffit pas pour traduire une victoire militaire en règlement politique favorable (à la fin, il faut aller au sol).

\section{Les usages, un autre point d'entrée, particulièrement riche pour s'approprier les imaginaires}

Une fois le contexte des imaginaires étudié, se pose la question de l'analyse des usages qu'ils permettent. Il s'agit ici de s'attacher à leurs modalités concrètes, voire parfois à l'usabilité, étant donné que les scénarios fictionnels sont en partie modelés par des contraintes de créativité.

L'examen de la production des œuvres de fictions montre que tous les choix en matière de «design» des objets et des interactions répondent à des logiques très différentes. Du réemploi d'éléments de décors disponibles - à l'instar des signalétiques lumineuses si caractéristiques du Los Angeles de Blade Runner - aux citations et références - courantes en matière de véhicule volant - voire enfin le grossissement de traits pour renforcer des effets dramatiques; il ne manque pas d'exemples d'interactions qui échappent à des objectifs de vraisemblance. Parfois, comme dans le cas de Ghost in the Shell, l'auteur apporte ce type de commentaire en marge d'une édition de l'ouvrage. Ceux-ci nous éclairent sur les procédés scénaristiques mis en œuvre et, surtout, sur les différentes ressources mobilisées pour « construire des mondes » en toute cohérence.

8 C'est le cas, par exemple, dans bien des vidéos de présentation d'équipements militaires futurs. Par exemple: https://www.youtube.com/watch?v=TDZkN08rA0Q;

${ }^{9}$ Général M. Yakovleff, conférence "Strategic Landpower for the XXI ${ }^{\text {st }}$ Century", Eurosatory/Ifri, 14 juin 2018 (disponible en vidéo sur YouTube). 


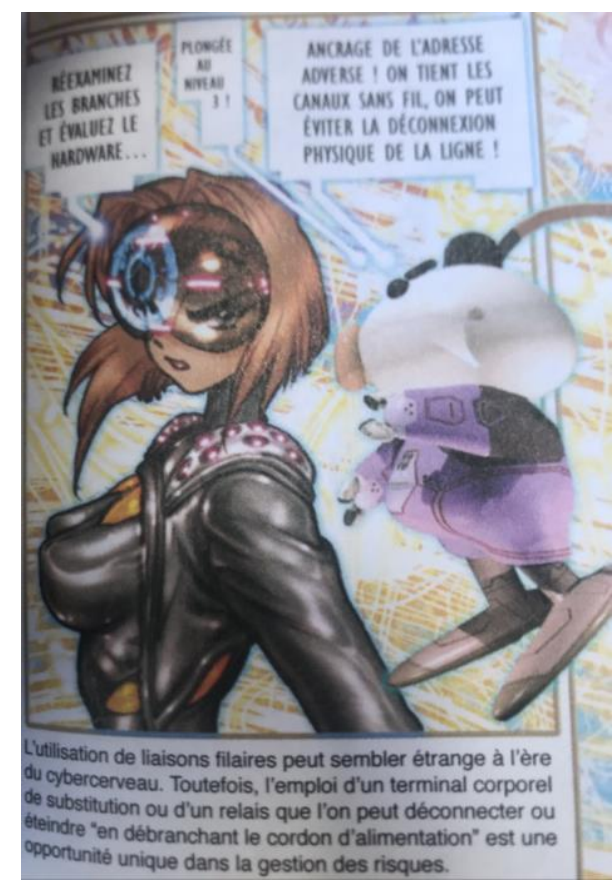

Figure 3. Ghost in the shell

Extrait de Ghost in the Shell - les IA accompagnant l'hérö̈ne devraient évidemment se trouver dans son champ de vision, et son casque ne peut pas être illuminé en bleu pour éviter d'être détectée. Pour autant, cette scénarisation est nécessaire pour embarquer le lecteur.

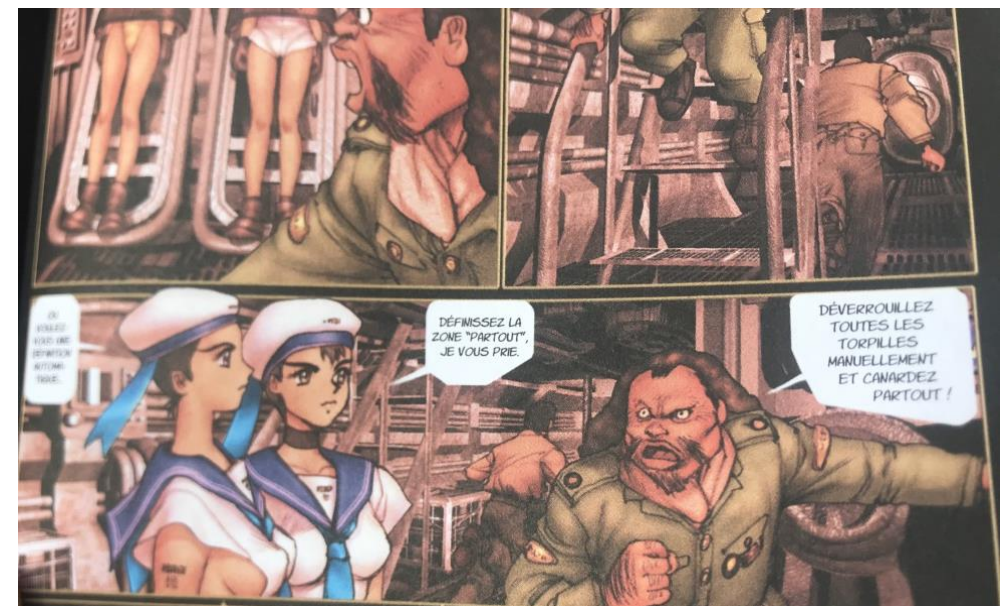

Figure 4. Ghost in the shell

Extrait de Ghost in the Shell - l'interaction entre le capitaine du sous-marin et les deux androïdes apporte peu de choses au scénario, mais est extrêmement réaliste en termes de cas d'usage car elle souligne la difficulté qu'il peut y avoir à donner des ordres à une IA lors d'un moment de stress.

Faut-il dès lors se limiter à la collecte d'imaginaires qui ont été élaborés dans des conditions de vraisemblance forte, à la façon des romans de « hard science fiction » nommés de la sorte en raison de l'intention de leurs auteurs de créer des récits les plus plausibles possible scientifiquement ? Nous pensons que non, dès lors que ces imaginaires ne sont pas uniquement élaborés dans une perspective de tester le futur. 
À titre d'exemple, prenons une série d'interactions très connues : celles que l'on retrouve de temps en temps dans la saga Star Wars : Han Solo aux commandes du poste de tir, essayant d'atteindre les vaisseaux ennemis.

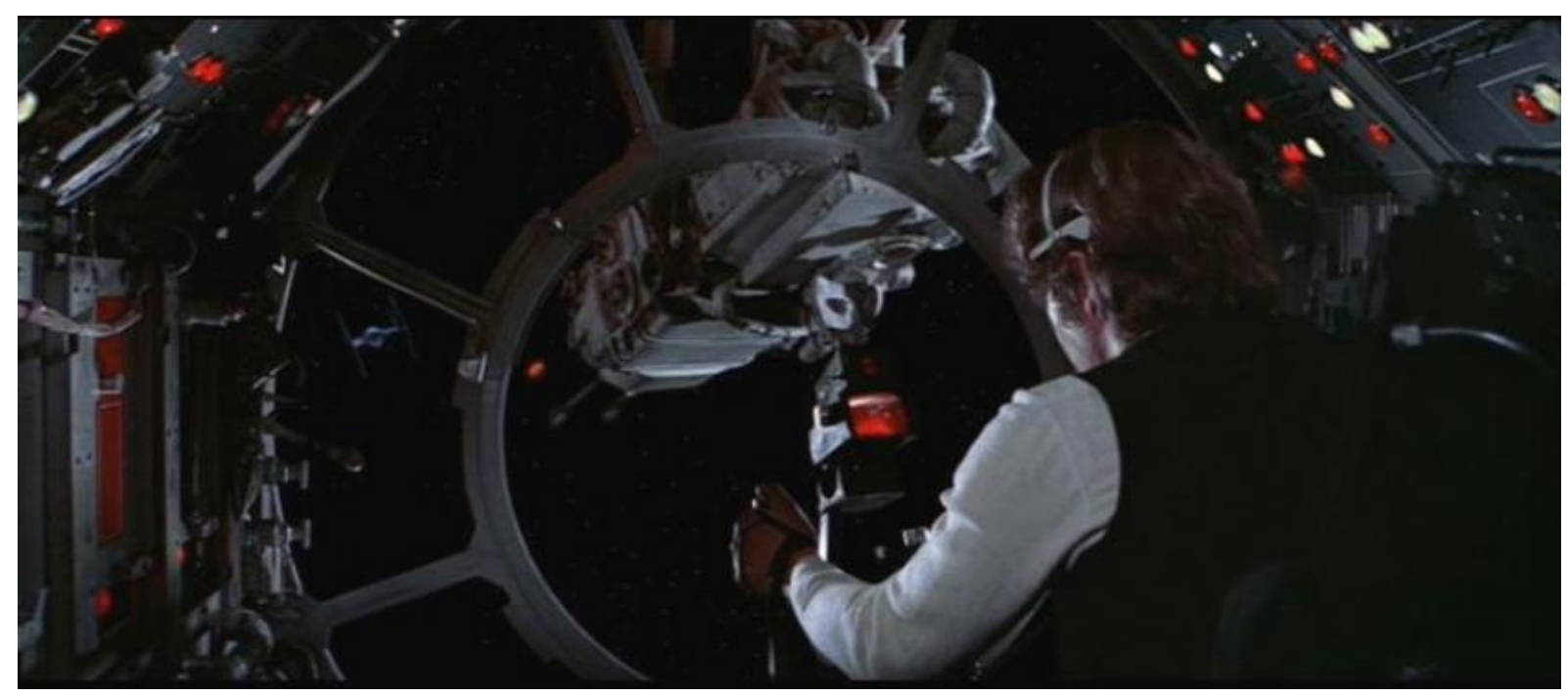

Figure 5. Han Solo tirant sur un vaisseau ennemi (Star Wars)

Pour ce faire, il utilise un écran avec deux cibles qu'il essaie de juxtaposer. On comprend, lorsque c'est le cas, qu'il est dans une situation de tir idéale, et qu'à priori, l'un des carrés le représente, l'autre carré représentant l'ennemi. Mais alors, si l'ordinateur de bord est en mesure d'analyser totalement une conduite de tir, pourquoi ne pas laisser la main à la machine ?

L'armure des protagonistes de l'ouvrage Iron Dragoons de Richard Fox, par exemple, le permet : «Gideon envoya un rocher en l'air. La main de Roland s'ouvrit et le rocher atterrit dans ses mains. "Bien, ton cerveau provoque l'impulsion, et l'armure fait la plupart des calculs" », décrit l'entraîneur du héros Gideon. Dans l'exemple de Star Wars, volontairement caricatural, on comprend, sur le plan narratif, l'importance d'avoir un Han Solo qui s'agite dans son poste de tir. La résolution du problème d'usage est alors plutôt évidente, les conduites de tir automatisées existant déjà pour les Close In Weapon Systems (CIWS) de nombreux navires de guerre. Bref, il s'agit d'une astuce narrative pour rendre la scène plus excitante. On peut toutefois se poser la question de l'utilité fonctionnelle d'une telle interaction. Éviter d'être piégé par des leurres non détectables par les machines ? Contribuer à la motivation psychologique du pilote lors de voyages sur de très longues distances à la fois éprouvants et monotones, etc. L'erreur - qui n'est alors qu'une question de point de vue et de logique de conception devient ici un stimulant sur le plan créatif.

Peut-on réellement parler de prédictions ratées ? Non, plutôt des limites de certaines projections, qui montrent assez rapidement la force de l'usage au regard des aspects technologiques. Or, les fictions ont tendance à projeter l'audience dans un univers de plausibilité technique, en se souciant moins de la valeur de l'usage. Chercher les aberrations de ce genre, c'est donc mettre en avant le rôle du banal, essentiel dans la vie des objets et de l'usage qui en sera fait. Concrètement, un certain nombre de produits imaginés ne sont tout simplement pas viables parce que leur usage réel les disqualifie aussitôt.

Les œuvres de fiction semblent parfois avoir conscience de ces limites, oscillant dans une même séquence entre des pôles très technologiques et d'autres plus « traditionnels » mais non moins efficaces.

En étudiant des écarts de ce type, et en montrant systématiquement les moyens plus simples de faire vivre une interaction dans un contexte au futur, l'erreur devient un moyen très utile pour identifier ce qui semble essentiel et robuste dans les usages possibles. Une façon d'apprivoiser la technologie, en 
particulier lorsque celle-ci est projetée dans des discours parfois très emphatiques quant à leur capacité à changer le futur.

Les personnages de fiction ont à leur disposition des armes très diverses, allant du laser à la manipulation mentale. Pour être réalistes, ces équipements, parfois fantastiques, doivent décrire avec force détails leurs interactions avec l'humain. C'est une matière très riche, notamment en termes d'usages et d'ergonomie physique et cognitive. Dans une scène de Kill Command, un sniper équipé de lentilles de vue connectées voit ce que filme l'optique de son fusil. Cela lui permet de se cacher derrière un muret, d'y poser son arme, et de tirer sans avoir à exposer sa tête.

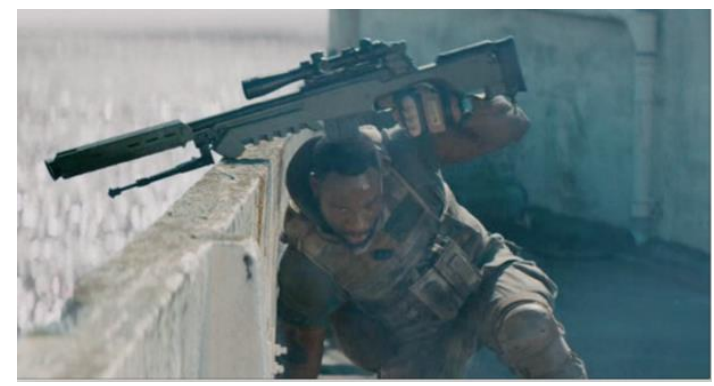

Figure 6. Kill command

Dans le film Spiderman Homecoming (2017), l'homme-araignée porte une combinaison offrant pas moins de huit cents options de tir. Pour s'y retrouver, une vision « tête haute » offre en surimpression la possibilité de sélectionner directement avec ses doigts le type de tir souhaité. Mais l'usage de l'interface est laborieux car le nombre de possibilités offertes est démesuré et le héros doit regarder ses mains pour procéder au choix.

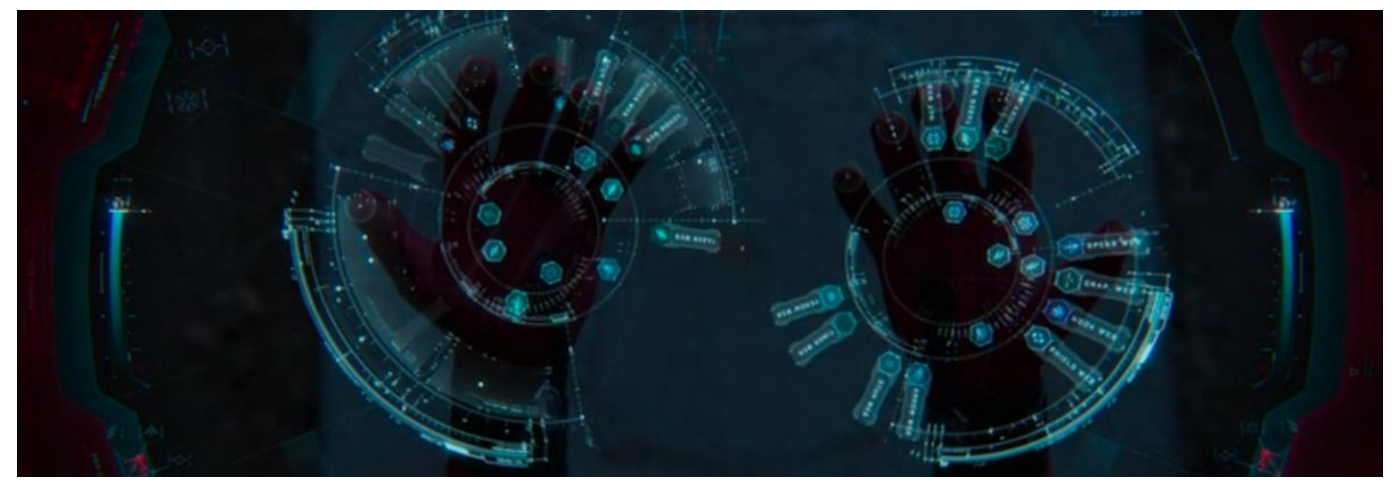

Figure 7. Spiderman Homecoming

Ce contre-exemple invite le spectateur à se poser la question de ce qui pourrait être intelligemment déporté sur les mains ou les avant-bras. En situation de stress, l'intuitivité du système est impérative. Or, ce n'est pas toujours le cas pour les équipements actuels comme les systèmes numérisés : «C'est leur manque flagrant de convivialité ainsi que leur rigidité qui rebutent le plus les utilisateurs, habitués à jongler avec aisance avec la numérisation civile, internet, le chat, l'envoi de SMS ou de pièces jointes » [JAC 13]. L'ergonomie et l'usage sont une préoccupation permanente aujourd'hui dans l'industrie de la défense. Il faut dire que plusieurs programmes d'armement ont eu à souffrir de problèmes dans ce domaine. C'est l'une des priorités de la dernière version de FELIN, la 1.3 : redonner de l'agilité au combattant débarqué ${ }^{10}$. Le système FELIN futur cherchera, lui, à mieux prendre en compte les besoins fondamentaux du combattant : communiquer, observer, tirer, se repérer, se protéger. Il devrait intégrer la réalité augmentée, des capacités de « décamouflage », géolocalisation indoor, vêtement de protection

\footnotetext{
${ }^{10}$ La lourdeur de la phase de préparation de la mission n'encourage pas à l'utilisation en entraînement. Cette version introduit le concept de «kit mission» permettant d'adapter l'équipement du combattant à la mission. Ce standard équipe les forces françaises depuis 2 ans. 
plus légers et probablement plus furtifs, objets connectés, progrès dans le domaine de l'énergie, etc. [NOI 18].

La gestion de l'information et de la communication du combattant apparait comme une autre ressource clef. La numérisation du champ de bataille, à l'œuvre depuis plusieurs années, avait été préalablement imaginée et «testée » dans les fictions. Briaros, le cyborg d'Apple Seed, est ainsi équipé de «grandes oreilles » qui lui permettent de se cacher derrière un pan de mur et de ne laisser dépasser qu'une caméra. Le flux vidéo généré peut ensuite être partagé avec ses équipiers et utilisé pour guider des tirs. Ce type d'approche est extrêmement bien développé dans les jeux vidéo de type First Person Shooter. On y retrouve de manière quasi systématique et ultra réalistes des cartographies de l'environnement, avec l'affichage des ennemis et amis, ainsi que d'éventuelles propositions de déplacement.

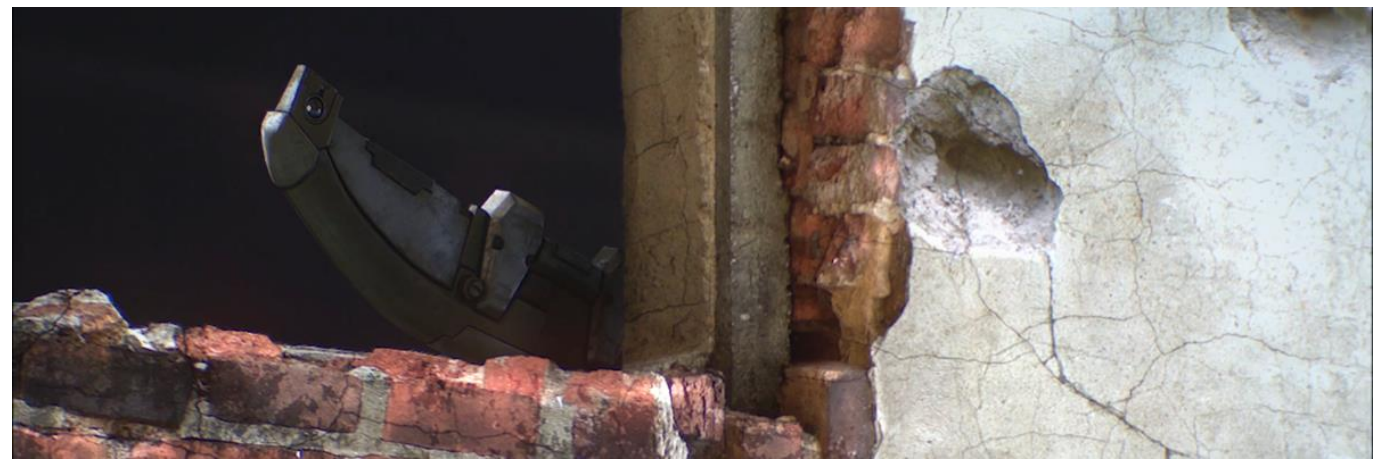

Figure 8. Apple Seed "des oreilles pour voir "

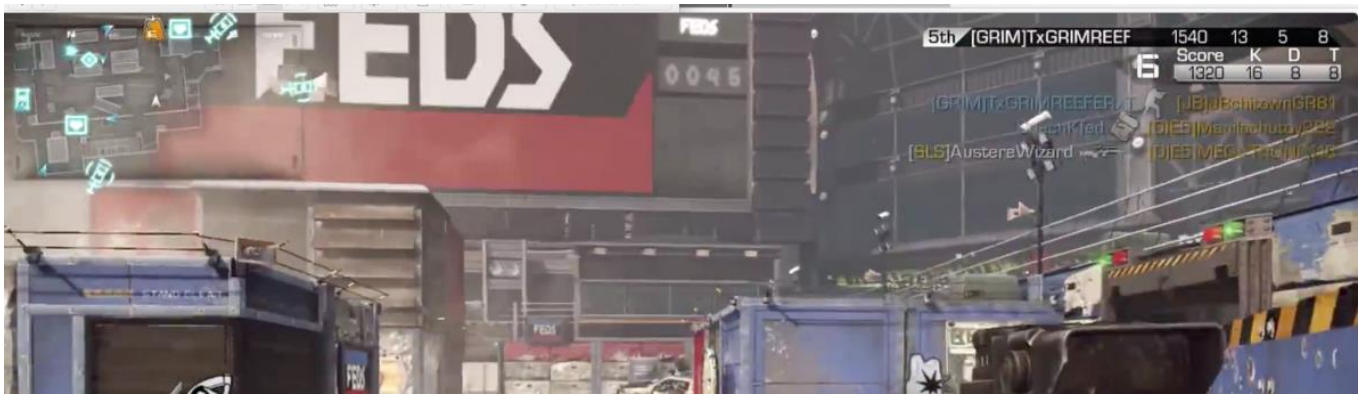

Figure 9. Cartographie tête haute dans Call of Duty

Ici aussi, la valeur de certains imaginaires réside surtout dans leur capacité à attirer l'attention sur les limites des technologies. Si le recours à l'hologramme pour la communication est visuellement très attractif dans une fiction, ses applications réelles montrent de nombreuses restrictions. Dans le jeu vidéo Batman Arkham Knight (2015), le héros appelle ses partenaires grâce à une projection sur son poignet. Batman est donc obligé d'interrompre son action et de focaliser son champ visuel sur son bras pour parler. Cela se traduit par une déconnexion dangereuse avec son environnement et le combat en cours, illustrant, sans le vouloir, les risques de la gestion de l'attention liée aux usages de la réalité augmentée. A l'inverse, une technologie permettant de représenter un environnement en amont des opérations peut être extrêmement utile pour préparer les missions. Un outil de ce type est présenté dans le film Black Panther (2018) où le héros, T'challa, utilise une table au-dessus de laquelle se matérialise le convoi qu'il doit attaquer. Il peut tourner autour, échanger avec sa garde du corps, et même interagir avec des composants virtuels en les prenant en main. Des technologies de ce type, sortes de « caisses à sable 2.0. » sont envisagées pour modéliser le champ de bataille [BER18]. 


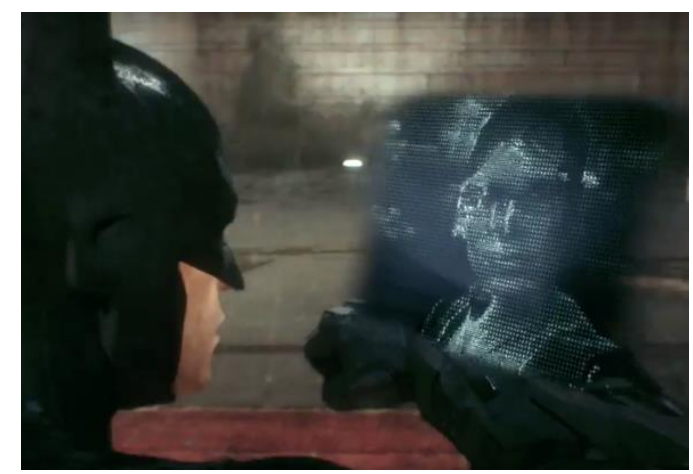

Figure 10. Arkham Knight : un hologramme pour communiquer

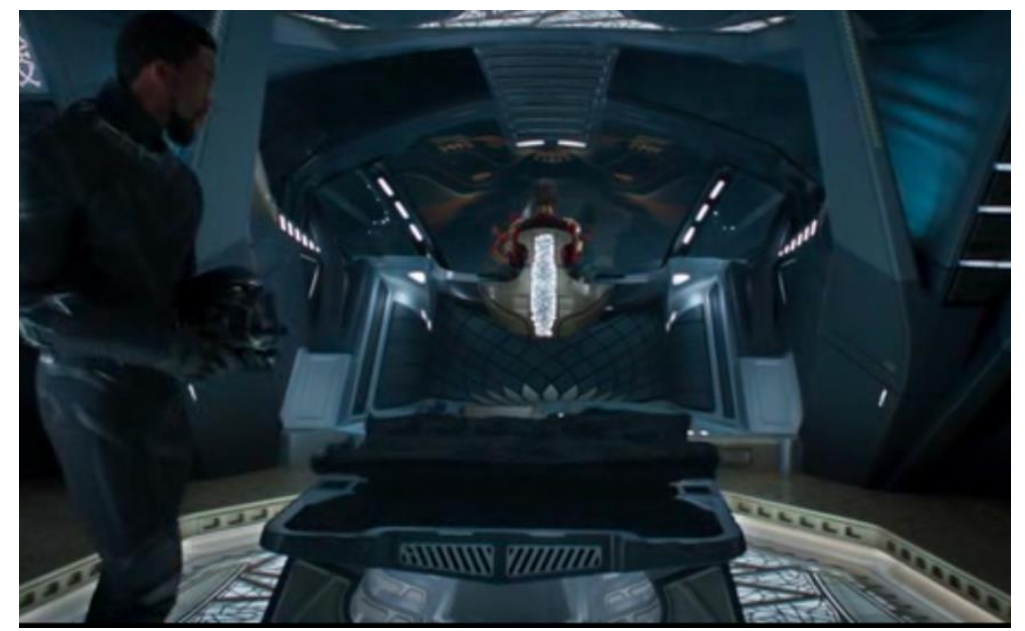

Figure 11. Black Panther : une visualisation 3D dynamique du terrain

Ces derniers exemples montrent un troisième type d'enjeu : les interactions du combattant avec son écosystème. Les jeux vidéo et certains films permettent ainsi de tester des collaborations nouvelles, notamment avec des drones ou des robots. Dans le film Elysium (2013) l'agent Kruger, pour tenter d'échapper à un poursuivant, lâche un essaim de mini drones situés dans des capsules sur sa main gauche et les dirige grâce à un pointeur laser qu'il tient dans sa main droite. Une fois qu'ils ont atteint leur cible, Kruger déclenche leur explosion en utilisant l'interface du poignet droit. Les détails de cette collaboration homme-machine sont très instructifs, or c'est un enjeu clef. Il s'agit en effet d'intégrer efficacement soldats, robots et intelligence artificielle dans un système de combat. Le gain espéré repose sur une complémentarité des forces de l'homme et de la machine. Par exemple, les systèmes automatisés dépassent de plus en plus les capacités humaines lorsqu'il s'agit d'activités codifiées et qui ont des objectifs clairs. Mais les algorithmes éprouvent des difficultés à « décider » face à l'incertitude et à l'ambiguïté des données ${ }^{11}$. A l'instar de ce que montre l'emploi des mules robotiques dans le film Spectral (2016), le schéma privilégié pour envisager le robot de l'avenir n'est pas, dans un premier temps, l'autonomie totale. C'est sa collaboration avec le combattant - chacun protégeant l'autre et «se répartissant le travail » - qui pourrait apporter une réelle plus-value opérationnelle.

\footnotetext{
${ }^{11}$ Joint Concept Note 1/18, "Human-Machine Teaming”, Ministry of Defence, mai 2018. 


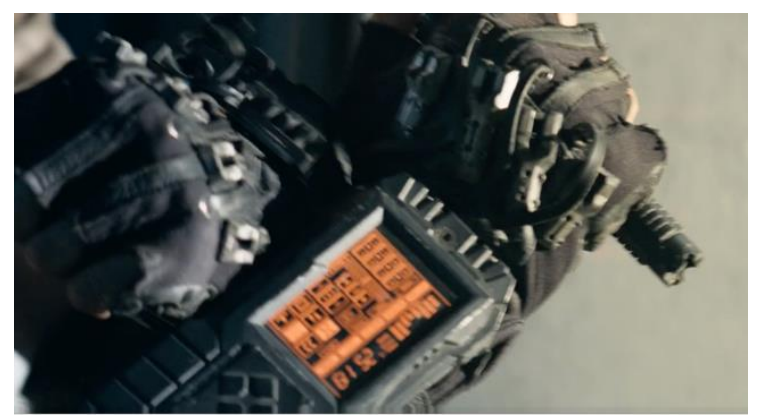

Figure 12. Elysium : le pointeur laser et l'essai de drones

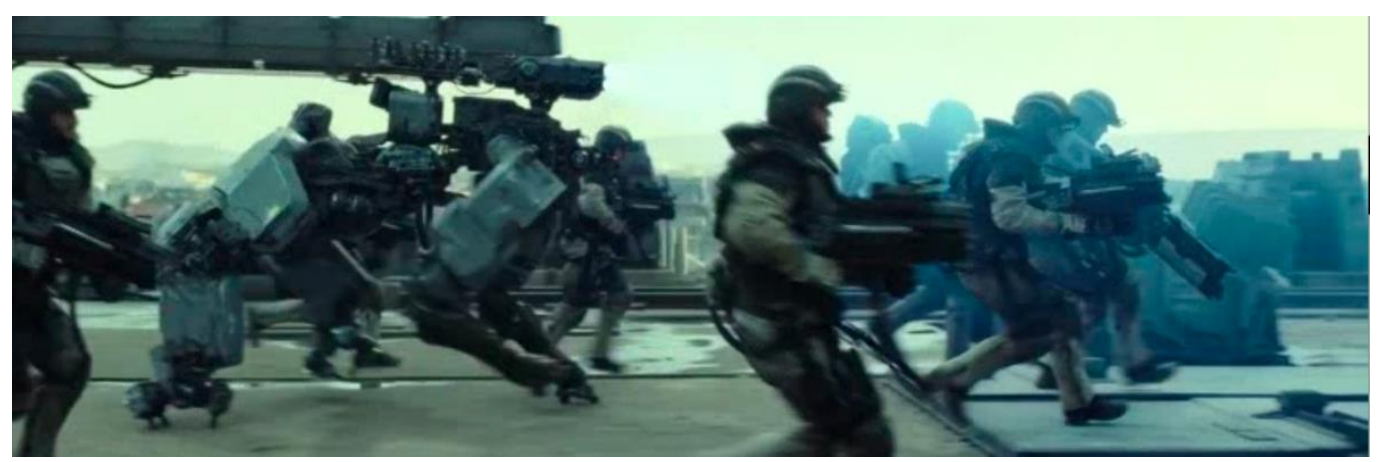

Figure 13. Spectral : utilisation d'une mule robotique

\section{Conclusion}

$\mathrm{Au}$ travers de ces différents niveaux d'analyses - identification des technologies mobilisées au sein d'un ensemble thématique d'imaginaires ; analyse des risques rendus lisibles par les mises en scenarios et enfin description des interactions concrètes - nous espérons avoir montré à quel point les imaginaires sont une source particulièrement féconde pour réfléchir à des enjeux opérationnels concrets (en particulier, dans cet article, nous avons traité des choix dans le développement de technologies d'augmentation; de l'équilibre entre analogique et numérique dans l'équipement du fantassin; du développement d'interfaces de visée ; de la gestion de l'information sur un champs de bataille et des capacités d'interaction avec les autres membres de sa compagnie). Ils ne sont pas générés de manières aléatoires, et à ce titre, sont un laboratoire pour imaginer, tester et projeter de futurs développements, ce qui nécessite de poursuivre dans la voie ici ouverte.

Quelques indices montrent que, dans le domaine de l'armée comme dans celui de la conception en général, cette circulation des idées est d'ores et déjà à l'œuvre, l'économie du spectacle et des loisirs s'appuyant sur des consultants des secteurs concernés pour formuler des propositions vraisemblables ; et les secteurs en questions mobilisant de manière explicite ou tacite - laissé au quant à soi des designers et ingénieurs et de leurs propres pratiques de fans amateur - ces ressources pour s'inspirer ou guider leurs réflexions.

Dans le premier cas, plusieurs travaux se sont attachés à documenter les liens entre cinéma, par exemple, et science [KIR 03] [KIR 11]. Des films réputés pour leur « réalisme » et capacité d'anticipation tels que 2001, l'odyssée de l'espace ont été élaborés en s'appuyant sur de très nombreux consultants d'entreprises. Les équipes d'IBM ont ainsi été mises à contribution dans la définition du fonctionnement de la célèbre intelligence artificielle. Dans un pamphlet consacré aux dangers du storytelling, Christian Salmon [SAL 07] illustre par de nombreux exemples les collaborations entre l'armée américaine et les auteurs hollywoodiens dans ce travail d'inspiration, ici pour créer des simulateurs des plus réalistes. 
Dans le second cas, à l'instar de ce que nous avons souligné dans le cas du Manga Ghost in the Shell, les dessinateurs et scénaristes peuvent être amenés à pousser très loin leurs spéculations quant à la vraisemblance de formes ou d'interactions. Si, comme nous l'avons montré plus haut, les enjeux de mise en scène l'emportent parfois sur la véracité - par exemple lorsque Masamune Shiro représente des torpilles équipées d'un système de brouillage, il ne dessine pas cet effet visuel pour éviter de "rendre le dessin incompréhensible », et il ne fait donc qu'en parler en notes de bas de page. Ailleurs, en revanche, on est saisi par le souci de détail et de réalisme de certaines images, élaborées sur la base d'une réflexion poussée à propos de la vraisemblance de la scène. Dans un exemple parmi d'autres, l'auteur explique ainsi que: "Chroma est un terminal humanoïde téléguidé, elle n'a donc pas besoin de fermer les yeux quand elle reçoit un choc ou d'expirer quand elle balance un coup de pied. Elle imite simplement les réactions de son pilote et ses mouvements ne veulent rien dire pour elle. » ${ }^{12}$ De même, dans la postface de la réédition récente de Le $4^{\text {ème }}$ pouvoir du dessinateur argentin Juan Rimenez, Christophe Quillien se pose la question «dessinateur ou ingénieur? » tant l'auteur pousse le détail technologique dans son travail. Ce dernier explique d'ailleurs :

« J'ai voulu montrer un environnement et des décors dans lesquels prédominent une technologie fonctionnelle, avec un maximum de détails. Je me fais toujours plaisir en dessinant, et je passe beaucoup de temps à ajouter des détails accessoires à mes vaisseaux, même si ils apparaissent à peine dans une scène $»^{13}$.

Les croquis qui accompagnent cette réédition montrent notamment comment des hypothèses sur l'agencement des pièces des moteurs de véhicules expliquent la forme visible de leur carrosserie. Loin de constituer des détails esthétiques, ils sont l'expression d'une réflexion préalable sur l'ingénierie fonctionnelle des machines.

Cette vigilance, exceptionnelle par son niveau de détail, n'est pas isolée au sein de la science-fiction. A l'instar de Sid Mead ${ }^{14}$, célèbre concepteur de la voiture volante du film Blade Runner, entre autres, il s'agit de se penser comme un «designer » ou un «ingénieur» devant résoudre des problèmes réels. Cette volonté de spéculer en fonction de contraintes fortes - celles imposées par le monde fictionnel sans compromission à un style qui sait également être "futuriste», est un levier créatif qui a pu générer des réalisations marquantes par leur vraisemblance. A plus forte raison lorsqu'il y a une véritable porosité professionnelle, un designer de film pouvant intervenir comme concepteur de véhicule (Sid Mead avait une pratique de design industriel, et il a aussi collaboré avec Raymond Lowy, par exemple, sur un projet de voiture soviétique) ou, plus simplement encore comme designer de jeux vidéo (ex. de Bradley Munkowitz concepteur de jeux pour Sony et des interfaces écrans de films tels que Tron et Oblivion). Les interviews plus récentes de designers de films convergent dans ce sens ${ }^{15}$.

Ces imaginaires sont des expériences parfois très poussées au niveau des possibilités de conception d'objets et de situations probables. Pourtant, les outils actuels pour produire et exploiter des collections d'imaginaires sont en grande partie laissés au quant à soi des personnes intéressées par ces démarches. L'absence de protocole formalisé va de pair avec l'absence de critères d'opérationnalisation de ces approches. Dans cet article, nous esquissons quelques axes de travail pour avancer dans cette direction. D'abord, en proposant de distinguer le type de connaissance exploitable (contexte VS interaction); ensuite en valorisant les grilles de lectures élaborées sur une analyse des échecs ; enfin en envisageant d'exploiter ces imaginaires comme des points de départ narratifs et non comme une «archéologie du futur » qu'il faudrait découvrir. L'enjeu consiste à dépasser une grille de lecture historique ou esthétique pour prendre en compte l'impact de la situation de réception d'une œuvre dans un contexte d'innovation concrète.

\footnotetext{
${ }^{12}$ Explication en note de bas de page de [SHI 01]

${ }^{13}$ Rimenez J., Le quatrième pouvoir, Les Humanoïdes Associés, 2018, p. 254.

${ }^{14}$ Interview publiée dans «Petite histoire du Spinner », Rockyrama hors série « Blade Runner », 2017.

${ }^{15}$ Voir, par exemple, l'excellente collection d'interviews sur le blog http://www.inventinginteractive.com/category/future/ 
Dès lors, nous suggérons qu'un champ de recherche pertinent porte sur l'identification des critères qui rendent possible l'appropriation des imaginaires, autrement dit la capacité à (1) générer des projections inédites au regard des thèmes récurrents au sein d'une communauté d'acteurs ; ensuite (2) la capacité à créer de nouveaux paradigmes face à une problématique donnée. Le tout en faisant en sorte que des participants non experts soient en mesure de s'approprier lesdits imaginaires.

L'approche que nous avons développée ici consiste à exploiter les imaginaires comme des points de départ pour tester les potentialités d'une conception. Or, tous n'ont pas la même pertinence. Parfois, clôt et lié à un monde particulièrement cohérent, l'imaginaire est difficilement transférable dans un environnement similaire au temps présent et une vision de futur proche crédible. Il est dès lors difficilement manipulable. A l'inverse, élusif et reposant sur des effets d'esthétique - à l'instar des évocations de véhicules volants sur Coruscant (Star Wars, épisode 1) - ils ne peuvent servir de test critique pour de possibles futurs. Entre les deux, il existe une énorme diversité d'imaginaires qui constituent des matériaux créatifs pertinents en tant que point de départ pour concevoir ou comme ressources pour tester et mettre en critique des propositions.

Nous faisons l'hypothèse que c'est dans la tension entre la focale proposée par l'imaginaire pour se montrer crédible vis à vis de son auditoire et ce qu'il nous offre comme espace de créativité (puisqu'il ne peut pas prendre en compte l'ensemble des contraintes du monde réel) que réside la capacité des imaginaires à constituer un laboratoire pour projeter des visions d'avenir crédibles et pertinentes. Un laboratoire dont l'exemple du fantassin que nous avons proposé montre, nous croyons, la pleine pertinence.

\section{Bibliographie}

[ATH 18] ATHERTON K. «Pentagon suspends commercial drone purchases and use », C4ISR.net, 13 juin 2018.

[AUG 13] AUGE J. «Speculative design : crafting the speculation », Digital Creativity, vol. 24, n 1, p. 11-35, 2013

[BER18] BERGOUNHOUX J., « Airbus modernise les briefings de l'armée de Terre », L'usine digitale, 15 juin 2018.

[COK 15] COKER, C., Future War, Polity, Londres, 2015

[COU 18] Coulton, P., Lindley, J., CoOPER, R. The little book of design fiction for the Internet of things, Petras, Lancaster, 2018.

[GOY 16] GOYA M., « Du bon usage du soldat augmenté », Inflexions, n³2, p.93-106, 2016.

[GRO 08] GRossman D. On Combat, Back Bay Books, Londres, 2008.

[HUB 00] HubIN G., Perspectives tactiques, Economica, Paris, 2000.

[JAC 13] JACOPS Y., «La NEB: limites et plus-values », Doctrine tactique, n²7, 2013

[KIR 03] KIRBY D., «Science Consultants, Fictional Films, and Scientific Practice », Social Studies of Science, vol. 33, $\mathrm{n}^{\circ} 2$, p. 231-268, 2003.

[KIR 11] KIRBY D., Lab Coats in Hollywood. Scientists impact on cinema, cinema's impact on sciences and technology, MIT Press, Cambridge, 2011.

[MIN 15] Minvielle, N., Wathelet, O., Masson, A. Jouer avec les futurs. Utilisez le design fiction pour faire pivoter votre entreprise, Pearson, Pearson, 2015.

[NOI 18] NOIZET T., «Que devrait être l'infanterie en 2038 ? », Fantassins magazine, n40, p.105-114, 2018

[RAS 08] RASMUSSEN N. On speed. The many lives of amphetamine, New York University Press, New York, 2008.

[SAL 07] SALMON C., Storytelling. La machine à fabriquer des histoires et à formater les esprits, Paris, La découverte, 2007.

[SHI 01] SHIROw M., Ghost In the Shell 2. Man Machine Interface, Glénat, Grenoble, 2001.

[ShU 06] Shuring D., Mock J.J., Justice B.J., Cummer S.A., Pendry J.B., StarR A.F., Smith D.R., « Metamaterial Electromagnetic Cloak at Microwave Frequencies », Science, vol. 314, n 5801, p.977-980, 2006. 
[SIN 15] P. Singer P., Cole A., Ghost Fleet, Eamon Dolan, New York, 2015. 